\title{
Direct Anterior Approach for Total Hip Arthroplasty in the Lateral Decubitus Position Versus Supine Position: A Random Clinical Trial
}

\section{Yao Xiao}

Fuzhou Second Hospital Affiliated to Xiamen University

\section{Zhanglai Li}

Fuzhou second Hospital Affiliated to Xiamen University

Feitai Lin

Fuzhou Second Hospital Affiliated to Xiamen University

Yiyuan Zhang

Fuzhou First Hospital Affiliated to Xiamen University

\section{Yan Weng}

Fuzhou Second Hospital Affiliated to Xiamen University

\section{Jinhua Chen}

Fujian Medical University Union Hospital

\section{Eryou Feng ( $\nabla$ fey9001@126.com )}

Fuzhou Second Hospital Affiliated to Xiamen University

\section{Research Article}

Keywords: Direct anterior approach, total hip arthroplasty, position

Posted Date: May 26th, 2021

DOl: https://doi.org/10.21203/rs.3.rs-510964/v1

License: (c) (i) This work is licensed under a Creative Commons Attribution 4.0 International License. Read Full License 


\section{Abstract}

Background: Direct anterior approach (DAA®for total hip arthroplasty (THA囚could be performed either in the lateral decubitus position or supine position. However, there is an obvious absence of literature regarding the differences, which position may be more conducive to recovery, technically more demanding, associated with component malposition or more complications.

Methods: From Jan.1st, 2020 to oct.1st, 2020, 45 patients were recruited for primary unilateral THA using the DAA. In total, 27 patients (60\%) underwent THAs using the DAA in the supine position, and 18 patients $(40 \%)$ in the lateral decubitus position. All surgeries were performed by a high-volume surgeon. Technical information, clinical and radiographic outcomes, SF-12 and patient-reported outcomes such as WOMAC were evaluated. All the date were tested with Generalized Linear Mixed Models Analysis, GLM Repeated Measurement Analysis, Independent samples t-test or Pearson's chi-square test.

Results: There were no differences in Population characteristics before surgery. The operation time, length of stay and blood loss in the LP group and the SP group were no differences. The prosthesis of the two groups were in a good position. pre-operative and the first and third day after the surgery of CK-MB and $\mathrm{Hb}$,pre-operative and the last follow-up of HSS,WOMAC,UCLA,VAS,SF-12,and pre-operation and postoperation of Offset,FA and LLD, all the above indicators show no significant difference. And the incidence of complications in the lateral position was lower than that in the supine position.

Conclusion: Both THA via DAA in the lateral decubitus position and in the supine position produced excellent clinical outcomes. From the perspective of the occurrence of complications, we are more inclined to use the lateral position.

\section{Introduction}

Total hip arthroplasty (THA) was regarded as one of the most successful and reliable procedures owing to restore function and reduces pain, consequently improving the quality of daily life for patients endstage hip joint disease [1]. However, the choice of proper surgical approach for THA is still debate [2-3]. More recently, the direct anterior approach (DAA) was recommended due to less muscle damage, lower rate of dislocation and faster recovery [4-7].

The DAA was conventionally performed in supine position using Hana table or ordinary operation table allowed folded during operation. Nevertheless, the DAA could be performed in lateral decubitus position either. Melman et al. reported their first experiences with the DAA in lateral decubitus position in 172 patients, demonstrated promising clinical results [8]. Min Chen et al. also displayed their experiences and early results of DAA in the lateral decubitus position, demonstrated that it might be a valuable alternative for absence of special operation table and provided satisfactory clinical and radiographic outcomes [9].

However, there is an obvious absence of literature regarding the differences, the DAA in which position for THA may be more conducive to recovery, technically more demanding, associated with component 
malposition or more complications. Therefore, the aims of this study were to examine the clinical and radiographic outcomes and distinguish between the DAA for THA in the lateral decubitus position versus supine position.

\section{Materials And Methods}

A random clinical trial was designed at our hospital to compare the clinical and radiographic outcomes between the DAA in the lateral decubitus position versus supine position for primary unilateral THA with at least 6-month follow up. Patients were recruited with end-stage hip joint diseases and indicated to undergo THA. The exclusion criteria included severe obesity (patients whose body mass index (BMI) > $40 \mathrm{~kg} / \mathrm{m}^{2}$ ), previous hip procedures with retained hardware, severe deformity of femur, posterior acetabular deficiency, stiff hip, Crowe type III/IV developmental dysplasia of the hip, or patients who were intolerance of operation, or could not be follow-up. All eligible patients were given written informed consent before surgery.

For each patient, comprehensive physical examination was conducted and medical history was collected including the patient's demographic data. Length of surgical procedures, perioperative blood loss, muscle injury, intraoperative and postoperative complications, length of stay were accessed. Standard anteroposterior radiographs of the pelvis and lateral radiographs of the femur were obtained for all patients preoperative, immediate postoperative, and latest follow up to compare the differences of component position and different length of limb (LLD) between the two group. Moreover, multi-spectral CT was specially obtained to evaluated for component position and alignment, especially for acetabular anteversion [10-11]. The Harris Hip Score (HSS), The Western Ontario and McMaster Universities Osteoarthritis Index (WOMAC), the visual analogue scale (VAS) and the SF-12 were used to assess the clinical results of these procedures. Extraordinary $\llbracket$ self- administered patient-reported outcome questionnaire冈the university of California at Los Angeles shoulder rating scale (UCLA), and the relative pre-operative and post-operative angle, like Offset, femoral anteversion(FA),cup abduction and cup anteversion, were especially performed to compare the clinical results between these two groups.

All procedures were performed by a high-volume orthopedic surgeon (FEY) and never participated the follow-up. THA were conducted using DDA in the lateral decubitus or supine position using standard surgical procedure $[8,9,12]$. The clinical and radiographic outcomes were calculated and assessed by another well-trained orthopedic surgeon who was ignorant about this study.

\section{Surgical procedure}

The difference between the two groups was preoperative position placement, The surgical technique was as follows. Taking the anterior superior iliac spine as the bony landmark, an 8-12-cm incision was made downward and backward each $2 \mathrm{~cm}$ toward the direction of the fibular head. The tensor fascia lata was split, allowing access to the anterior hip capsule through the interval between rectus femoris and gluteus medius. Ligating and electrocoagulating the arteria circumflexa femoris lateralis ascending branch, and 
the anterior capsule of the joint was fully exposed. The femoral neck osteotomy was performed according to the preoperative measurement plan. Taking out the femoral head, and removing the adipose tissue of the acetabular labrum and fossa ovale under direct vision. After the acetabulum was exposed. Once the labrum and cotyloid fat are removed, reaming begins. The cup abduction was determined by the angle between the longitudinal axis of the body and acetabular reamers. The cup anteversion was determined by the angle between horizontal axis of bed and acetabular reamers. Then the appropriate size of acetabular prosthesis was implanted. Adjusting the bed, The upper and rear capsules of the joint were loosened to expose the proximal femur. femoral was reamed until get a suitable size. After a satisfactory trial, the femoral stem and femoral head prosthesis were implanted to test joint mobility.

\section{Statistics}

Test of homogeneity of variance and normal distribution was performed before statistical analysis. Pearson's chi-square test was conducted for normal variable parameters (sex and surgical site). Independent samples t-test was used for normal distributed variable parameters (age, BMI, operation time, length of stay, blood loss, component position, Cup Abduction, Cup Anteversion). Besides, hemoglobin, CK-MB, HSS, WOMAC, SF-12, Femoral anteversion and Offset values were tested with a GLM Repeated Measurement Analysis. The VAS and LLD were tested with Generalized Linear Mixed Models Analysis. Alpha of 0.05 was considered to be significant. Data are expressed as mean \pm SD. All statistical analyses were performed using IBM SPSS software for Version 20.0 (SPSS Inc, Chicago, IL)

\section{Result}

\section{Patient characteristics}

There where no statistical differences between age (56.891 yrs vs $57.521 \mathrm{yrs} ; \mathrm{p}=0.894)$ and $\mathrm{BMI}(23.329$

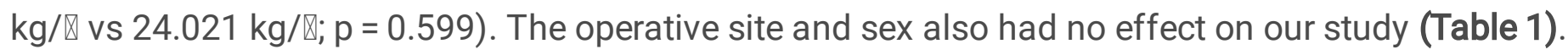

\section{Operative data}

After the surgery, operation time, length of stay and blood loss were recorded (Table 2). P values calculated by the three were all greater than 0.05 , showing no statistical significance.

\section{CK-MB and $\mathrm{Hb}$ values}

CK-MB and $\mathrm{Hb}$ values were recorded on the first and third day after the surgery and compared with preoperative values. The statistical results showed that no interaction exist in the two group between $\mathrm{Hb}$ value $(p=0.655)$ and CK-MB value $(p=0.873)$ (Table 3, Fig. 1, Fig. 2$)$, which means the $\mathrm{Hb}$ and CK-MB value were not significantly different in both surgical groups, and this further verified that there was no difference in blood loss as mentioned above. On the other hand, the difference of $\mathrm{Hb}$ value $(\mathrm{p}<0.001)$ and CK-MB value $(p<0.001)$ between the respective groups were statistically significant (Table 3, Fig. 1 , Fig. 2). As far as $\mathrm{Hb}$ value is concerned, surgical blood loss led to a gradual significant decrease. As for 
CK-MB value, operation led to a rapid significant increase in CK-MB value on the first day after surgery, while CK-MB value on the third day after surgery showed no obvious variation trend.

\section{Clinical score}

All patients had received the final follow-up and all the scores were calculated, The average final followup time is 10.910 months (4-14 months). There where no statistical differences between both groups regarding pre-operative and the last follow-up of $\operatorname{HSS}(p=0.141), \operatorname{WOMAC}(p=0.216), \operatorname{UCLA}(p=$ $0.669), \operatorname{VAS}(p=0.357)$ and SF-12(MCS = 0.434;PCS = 0.632)(Table 3). However, the scores of both groups were improved obviously, making a good clinical satisfactory result(Table 4 - 1).

\section{Radiographic outcomes}

Comparing the differences in Offset, FA and LLD of pre-operation and post-operation, and the postoperative cup abduction and anteversion, the further results are shown in Table 3 and Table 4 - 2. None of the data was statistically significant.

\section{Discussion}

A large amount of researches have been done on the comparison of direct anterior approach with other approaches, demonstrated that patients undergoing the DAA for THA had slightly shorter incision length and blood loss. Zhao Wang et al. researched a meta-analysis about 184 cases of DAA and 175 cases of PA, demonstrated that the average incision length of DAA for THA was $3.51 \mathrm{~cm}$ less than posterior approach [13]. Klasan et al. compared DAA with anterolateral approach, demonstrated that the blood transfusion rate was statistically decreased in DAA group, with $5.8 \%$ of $14.1 \%$ lower compared to anterolateral approach [14]. Whereas, DAA could be performed in lateral decubitus position or supine position, and there were few studies conducted on comparison these two different positions of DAA for THA.

The supine position is the position in which most surgeons begin to learn DAA, and it is also described in most studies, especially in the posterolateral position. Malahias et al. believe that simultaneous bilateral THA at the same time is effective and safe [15]. In order to reduce the operation time, lateral position should be avoided when the patient requires bilateral surgery. While the supine position can be placed once to complete the operation, which greatly saves the operation time. According to Guler et al, supine surgery is advantageous in terms of anesthesia [16]. Meanwhile, c-arm machine is more acceptable, the photography is more accurate, theoretically, it is easier to locate the anatomical position of the pelvis, and acetabular cup implantation is more accurate. In 1985, Robert and Judet et al used a traction bed during the DAA operation. Moreau et al. proposed that DAA was originally performed in the supine position on a specialized traction bed [17]. However, Camenzind et al. believed that the use of traction bed in the supine DAA greatly increased the incidence of postoperative complications. Therefore, 138 patients were selected to exprience THA with supine position DAA, and only 3 patients had complications, with an incidence of $2.1 \%$. Combining with the 24-months follow-up, it was believed that the DAA in supine position with trecion bed showed better early clinical efficacy [18]. Sarraj et al. compared DAA with use of 
a traction bed and a standard operating bed, and concluded that the standard operating bed and traction bed had similar clinical outcomes and complication rates. Using the standard operating bed had more advantages, including reduced blood loss, shorter operating time, and fewer intraoperative fractures rates [19]. Meanwhile, it also has disadvantages such as the need for more assistants, the difficulty in the femur exposing, and the possibility of additional soft tissue injury. Lovell et al. believe that DAA with a standard surgical bed has potential advantages, including the position of the operated limb doesn't need to adjust during the procedure, and it's easier to access hip stability, range of motion and limb length [20]. Therefore, there are few studies on the selection of surgical beds, which are mainly determined by the preference and habits of surgeons, as well as by the factors such as cost and technology.

As we know, the traditional position of the classic posterolateral surgery is lateral position. When the operative limb is in overextension, abduction and external rotation, it is more likely to expose the femur and enter the medullary space, which can reduce the incidence of complications, and in the lateral decubitus position, the abdominal adipose tissue can be removed from the incision and surgical field due to gravity, besides, Slotkin et al. suggested that the lateral position causes pelvic orientation changes that may affect the implantation of the acetabular prosthesis, whereas the supine position produces less pelvic orientation changes $[8,9,21]$.

Within our cohort, Operative data (like Operation time/Length of stay/CK-MB/Hb values) showed no statistical significance. This could be due to the reason that DAA is a minimally invasive surgery with small incision and using a muscle gap approach, which makes less injure and bleeding. Although showing no difference, we believe that it's relevant to the insufficient number of cases in the whole study. We understand In DAA no muscles were cut, but considerable traction had to be applied to obtain good exposure Some authors believe that in the lateral position, the soft tissue around the incision can make full use of the advantages of gravity to expose the surgical area better and reduce blood loss [22, 23]. Of course, we still need further research on whether lateral position can reduce blood loss and muscle injury.

Clinical scores also appear not to depend on position. There is no evidence that either lateral or supine position can recover faster or better. Although, on our study, no difference was showed in clinical scores between the two groups, all patients obtained significantly excellent functional scores and improved quality of life during the last follow-up.

Radiographic outcomes after surgery have been linked to clinical outcomes. We measured cup

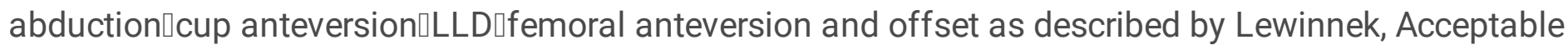
cup abduction was defined as between $30^{\circ}$ and $50^{\circ}$, acceptable cup anteversion angle was defined as between $5^{\circ}$ and $25^{\circ}$ [24]. In our study, postoperative radiological outcomes showed that the prosthesis of the two groups was in a good position. LLD value was equal to operative side minus the other side, which was denoted as positive if the operative extremity is longer than the contralateral side. In fact, some clinical articles didn't measure offset and LLD, while smaller offset was regarded as a significant risk factor for LFCN injury following THA via a DAA $[25,26]$. Although our findings showed radiographic outcomes were no statistical differences, generally each was improved by surgery. Nowadays, in terms of 
functional recovery, it has still not been demonstrated which radiographic date are most important, each parameter is meaningful.

The pelvic position is more reliable when in the supine position, leading to more consistent orientation of the acetabular component [27]. For two case of Ankylosing Spondylitis, we decide to choose the lateral position, because it is difficult for the patients to lie flat and anesthesia. The patient is in the lateral position, the exposure would be better for greater back extension and limb adduction [28].

We are all too familiar with the complications of DAA. Many clinicians have done research about complications of DAA. Comparing to the other approaches, DAA has the higher incidence of complications [29]. The most common complication after DAA was nerve dysfunction followed by intraoperative fractures, including intraoperative trochanter and femoral fractures, especially [30, 31]. Moreover, several studies have reported that complication rates of DAA may relate to the so-called "learning-curve period" for the surgeon After 50 or more procedures need to be performed, the rate of revision would reach a steady level [32]. In Chinese patients, analysis showed that complication rates and operating time normalize after 88 cases and 72 cases respectively [33]. However, there was no significant relationship between femoral nerve palsy and surgeon's experience of DAA [34]. Paul et al. also showed that using ceramic-on-ceramic implant through DAA made a good clinical results and implant survival rates at a mean follow-up of 31.9 months, 111 hips (96\%) were perceived no serous complication [35]. In our study, few complications happened after the operation. In the lateral position, only 1 case of dislocation occurred postoperatively. While in the supine position, 1 case of dislocation and 1 case of greater trochanter fracture occurred intra-operatively, 2 cases were due to difficult exposure during operation.; 1 case of unexplained recurrent fever, 1 case of dislocation and 1 case of poor hip flexion occurred after operation. Although it is theoretically considered that the dislocation rate of DAA is lower than other approaches, dislocation still occurred in both positions. For the case of intraoperative dislocation, we have taken to reduce the femoral anteversion and then dislocation didn't happen again. And we still experienced one case of greater trochanteric fracture occurring during the exposure process, which is considered to be caused by osteoporosis. Nevertheless, the results showed that the incidence of complications in the lateral position was lower than that in the supine position, but this result should be verified in a larger sample to ensure it is not due to inadequate statistical power.

Making a summary to sum up the above points. The lateral position has the following advantages:

1. Proximal femoral exposure is more convenient;

2. The incision exposure is more convenient for the reason that muscular adipose tissue won't hidden the incision;

3. Special traction bed and special surgical instruments are not required, it's no need to adjust intraoperative the operating bed intraoperatively;

4. For most surgeons who study the posterolateral approach firstly, the habit of acetabular process does not change; 
5. There is no need to change the position when posterior lateral incision is needed, in the case of greater trochanter fracture.

6. the incidence of complications are lower.

Disadvantages:

1. Pelvic fixation is needed before operation;

2. It is inconvenient to compare the length of bilateral limbs during the operation.

The supine position has the following advantages:

1. Simultaneous surgery can be performed on both sides without changing body position.

2. It is convenient to compare the length of bilateral limbs during the operation.

3. Facilitating intraoperative fluoroscopy, reducing the risk of anesthesia, increasing the safety of surgery.

4. In theory, acetabular prosthesis implantation is more accurate.

Disadvantages:

1. Special traction bed and special surgical instruments are needed.

2. Proximal femoral exposure is difficult and greater trochanteric fractures are more likely to happen.

\section{Conclusion}

Both THA via DAA in the lateral decubitus position and in the supine position produced excellent clinical outcomes. From the perspective of the occurrence of complications, we are more inclined to use the lateral position.

\section{Abbreviations}

DAA: Direct anterior approach,

THA: total hip arthroplasty,

LLD: length of limb,

HSS: The Harris Hip Score,

WOMAC: The Western Ontario and McMaster Universities Osteoarthritis Index,

VAS: the visual analogue scale,

UCLA: the university of California at Los Angeles shoulder rating scale, 
SF-12: The 12-item Short Form Survey,

FA : femoral anteversion

\section{Declaration}

\section{Ethics approval}

Approval was obtained from the ethics committee of Fuzhou Second Hospital Affiliated to Xiamen University. The procedures used in this study adhere to the tenets of the Declaration of Helsinki.

Consent to participate

Informed consent for all participants have been obtained,and identifying details of the participants will not be published in written descriptions.

Consent for publication

All authors declare that they agree to publish.

Availability of data and material

The datasets used or analysed during the current study are available from the corresponding author on reasonable request.

Competing interest

All authors certify that they have no affiliations with or involvement in any organization or entity with any financial interest or non-financial interest in the subject matter or materials discussed in this manuscript.

Funding

No funding was received to assist with the preparation of this manuscript.

Code availability

The statistical software used in this paper is the SPSS software 20.0.

Authors' contributions

All authors contributed to the study conception and design. Material preparation, data collection and analysis were performed by Yao Xiao and Feitai Lin. The first draft of the manuscript was written by Yao Xiao and Zhanglai Li,all authors commented on previous versions of the manuscript. All authors read and approved the final manuscript.

Acknowledgements 
Grateful acknowledgment is made to my supervisor, Professor Eryou Feng, who gave me considerable help by means of suggestion, comments and criticism. Without his pushing me ahead, the completion of this thesis would be impossible. In addition, I deeply appreciate the contribution to this thesis made in various ways by Zhanglai Li and Jinhua Chen.I also owe my sincere gratitude to my friends and my fellow classmates who. gave me their help and time in listening to me and helping me work outmy problems during the difficult course of the thesis.

\section{References}

1. Learmonth ID, Young C, Rorabeck C. The operation of the century: total hip replacement. Lancet. 2007,370(9597):1508-1519. https://doi.org/10.1016/S0140-6736(07)60457-7.

2. Alecci V, Valente M, Crucil M, Minerva M, Pellegrino CM, Sabbadini DD. Comparison of primary total hip replacements performed with a direct anterior approach versus the standard lateral approach: perioperative findings. J Orthop Traumatol. 2011,12(3):123-129. https://doi.org/10.1007/s10195011-0144-0.

3. Taunton MJ, Trousdale RT, Sierra RJ, Kaufman K, Pagnano MW. John Charnley Award: Randomized Clinical Trial of Direct Anterior and Miniposterior Approach THA: Which Provides Better Functional Recovery?. Clin Orthop Relat Res. 2018,476(2):216-229.

https://doi.org/10.1007/s11999.0000000000000112.

4. Bergin PF, Doppelt JD, Kephart CJ, et al. Comparison of minimally invasive direct anterior versus posterior total hip arthroplasty based on inflammation and muscle damage markers. J Bone Joint Surg Am. 2011,93(15):1392-1398. https://doi.org/10.2106/JBJS.J.00557.

5. Masonis JL, Bourne RB. Surgical approach, abductor function, and total hip arthroplasty dislocation. Clin Orthop Relat Res. 2002,(405):46-53. https://doi.org/10.1097/00003086-200212000-00006.

6. Hoell S, Sander M, Gosheger G, Ahrens H, Dieckmann R, Hauschild G. The minimal invasive direct anterior approach in combination with large heads in total hip arthroplasty - is dislocation still a major issue? a case control study. BMC Musculoskelet Disord. 2014,15:80. https://doi.org/10.1186/1471-2474-15-80.

7. Barrett WP, Turner SE, Leopold JP. Prospective randomized study of direct anterior vs postero-lateral approach for total hip arthroplasty. J Arthroplasty. 2013,28(9):1634-1638. https://doi.org/10.1016/j.arth.2013.01.034.

8. Melman WP, Mollen BP, Kollen BJ, Verheyen CC. First experiences with the direct anterior approach in lateral decubitus position: learning curve and 1 year complication rate. Hip Int. 2015,25(3):251-257. https://doi.org/10.5301/hipint.5000221.

9. Chen M, Luo Z, Ji X, Cheng P, Tang G, Shang X. Direct Anterior Approach for Total Hip Arthroplasty in the Lateral Decubitus Position: Our Experiences and Early Results. J Arthroplasty. 2017,32(1):131138. https://doi.org/10.1016/j.arth.2016.05.066.

10. Fujishiro T, Hayashi S, Kanzaki N, et al. Computed tomographic measurement of acetabular and femoral component version in total hip arthroplasty. Int Orthop. 2014,38(5):941-946. 
https://doi.org/10.1007/s00264-013-2264-z.

11. Loftus M, Ma Y, Ghelman B. Acetabular Version Measurement in Total Hip Arthroplasty: the Impact of Inclination and the Value of Multi-Planar CT Reformation. HSS J. 2015,11(1):65-70. https://doi.org/10.1007/s11420-014-9416-6.

12. Hartford JM, Bellino MJ. The learning curve for the direct anterior approach for total hip arthroplasty: a single surgeon's first 500 cases. Hip Int. 2017,27(5):483-488. https://doi.org/10.5301/hipint.5000488.

13. Wang Z, Hou JZ, Wu CH, et al. A systematic review and meta-analysis of direct anterior approach versus posterior approach in total hip arthroplasty. J Orthop Surg Res. 2018,13(1):229. https://doi.org/10.1186/s13018-018-0929-4.

14. Klasan A, Neri T, Oberkircher L, Malcherczyk D, Heyse TJ, Bliemel C. Complications after direct anterior versus Watson-Jones approach in total hip arthroplasty: results from a matched pair analysis on 1408 patients. BMC Musculoskelet Disord. 2019,20(1):77. https://doi.org/10.1186/s12891-019-2463-x.

15. Malahias MA, Chulsomlee K, Thorey F. Simultaneous bilateral minimally invasive total hip arthroplasty: A comprehensive review of the literature. Orthop Rev (Pavia). 2018,10(3):7677. https://doi.org/10.4081/or.2018.7677.

16. Güler O, Öztürk S, Özgezmez FT, Çerçi MH. Comparison of Supine and Lateral Decubitus Positions for Total Hip Arthroplasty with the Direct Lateral Approach in Overweight and Obese Patients. Biomed Res Int. 2020,2020:8684067. https://doi.org/10.1155/2020/8684067.

17. Moreau P. Minimally invasive total hip arthroplasty using Hueter's direct anterior approach. Eur J Orthop Surg Traumatol. 2018,28(5):771-779. https://doi.org/10.1007/s00590-018-2158-2.

18. Camenzind RS, Stoffel K, Lash NJ, Beck M. Direct anterior approach to the hip joint in the lateral decubitus position for joint replacement. Der ventrale Zugang zum Hüftgelenk in Seitenlage zur Implantation einer Hüfttotalendoprothese. Oper Orthop Traumatol. 2018,30(4):276-285. https://doi.org/10.1007/s00064-018-0550-z.

19. Sarraj M, Chen A, Ekhtiari S, Rubinger L. Traction table versus standard table total hip arthroplasty through the direct anterior approach: a systematic review. Hip Int. 2020,30(6):662-672. https://doi.org/10.1177/1120700019900987.

20. Lovell TP. Single-incision direct anterior approach for total hip arthroplasty using a standard operating table. J Arthroplasty. 2008 Oct,23(7 Suppl):64-8. https://doi.org/ 10.1016/j.arth.2008.06.027.

21. Slotkin EM, Patel PD, Suarez JC. Accuracy of Fluoroscopic Guided Acetabular Component Positioning During Direct Anterior Total Hip Arthroplasty. J Arthroplasty. 2015,30(9 Suppl):102-106. https://doi.org/10.1016/j.arth.2015.03.046.

22. Locher $S$, Kühne R, Lottenbach M, Bamert P. Blutverlust bei Hüfttotalprothesenimplantationen: Seitenlage versus Rückenlage [Blood loss in total hip prosthesis implantation: lateral versus supine position]. Z Orthop Ihre Grenzgeb. 1999,137(2):148-152. https://doi.org/10.1055/s-2008-1039350. 
23. Widman J, Isacson J. Lateral position reduces blood loss in hip replacement surgery: a prospective randomized study of 74 patients. Int Orthop. 2001,25(4):226-227. https://doi.org/10.1007/s002640100254.

24. Lewinnek GE, Lewis JL, Tarr R, Compere CL, Zimmerman JR. Dislocations after total hip-replacement arthroplasties. J Bone Joint Surg Am. 1978,60(2):217-220.

25. Lin TJ, Bendich I, Ha AS, Keeney BJ, Moschetti WE, Tomek IM. A Comparison of Radiographic Outcomes After Total Hip Arthroplasty Between the Posterior Approach and Direct Anterior Approach With Intraoperative Fluoroscopy. J Arthroplasty. 2017,32(2):616-623. https://doi.org/10.1016/j.arth.2016.07.046.

26. Ozaki Y, Homma Y, Sano K, et al. Small femoral offset is a risk factor for lateral femoral cutaneous nerve injury during total hip arthroplasty using a direct anterior approach. Orthop Traumatol Surg Res. 2016,102(8):1043-1047. https://doi.org/10.1016/j.otsr.2016.08.019.

27. Grammatopoulos G, Gofton W, Cochran M, et al. Pelvic positioning in the supine position leads to more consistent orientation of the acetabular component after total hip arthroplasty. Bone Joint J. 2018,100-B(10):1280-1288. https://doi.org/10.1302/0301-620X.100B10.BJJ-2018-0134.R1.

28. Wu H, Cheng WD, Jing J. Total hip arthroplasty by direct anterior approach in the lateral position for the treatment of ankylosed hips. Eur J Orthop Surg Traumatol. 2020,30(6):993-1001. https://doi.org/10.1007/s00590-020-02655-w.

29. Aggarwal VK, Elbuluk A, Dundon J, et al. Surgical approach significantly affects the complication rates associated with total hip arthroplasty. Bone Joint J. 2019,101-B(6):646-651. https://doi.org/10.1302/0301-620X.101B6.BJJ-2018-1474.R1.

30. Lee GC, Marconi D. Complications Following Direct Anterior Hip Procedures: Costs to Both Patients and Surgeons. J Arthroplasty. 2015,30(9 Suppl):98-101. https://doi.org/10.1016/j.arth.2015.03.043.

31. De Geest T, Fennema P, Lenaerts G, De Loore G. Adverse effects associated with the direct anterior approach for total hip arthroplasty: a Bayesian meta-analysis. Arch Orthop Trauma Surg. 2015,135(8):1183-1192. https://doi.org/10.1007/s00402-015-2258-y.

32. de Steiger RN, Lorimer M, Solomon M. What is the learning curve for the anterior approach for total hip arthroplasty?. Clin Orthop Relat Res. 2015,473(12):3860-3866. https://doi.org/10.1007/s11999015-4565-6.

33. Kong X, Grau L, Ong A, Yang C, Chai W. Adopting the direct anterior approach: experience and learning curve in a Chinese patient population. J Orthop Surg Res. 2019,14(1):218. https://doi.org/10.1186/s13018-019-1272-0.

34. Hoshino C, Koga D, Koyano G, et al. Femoral nerve palsy following primary total hip arthroplasty with the direct anterior approach. PLoS One. 2019,14(5):e0217068. https://doi.org/10.1371/journal.pone.0217068.

35. Henri Bauwens P, Fary C, Servien E, Lustig S, Batailler C. Early low complication rate of ceramic-onceramic total hip arthroplasty by direct anterior approach. SICOT J. 2020,6:30. https://doi.org/10.1051/sicotj/2020027. 


\section{Tables}

Due to technical limitations, tables are only available as a download in the Supplemental Files section.

Figures

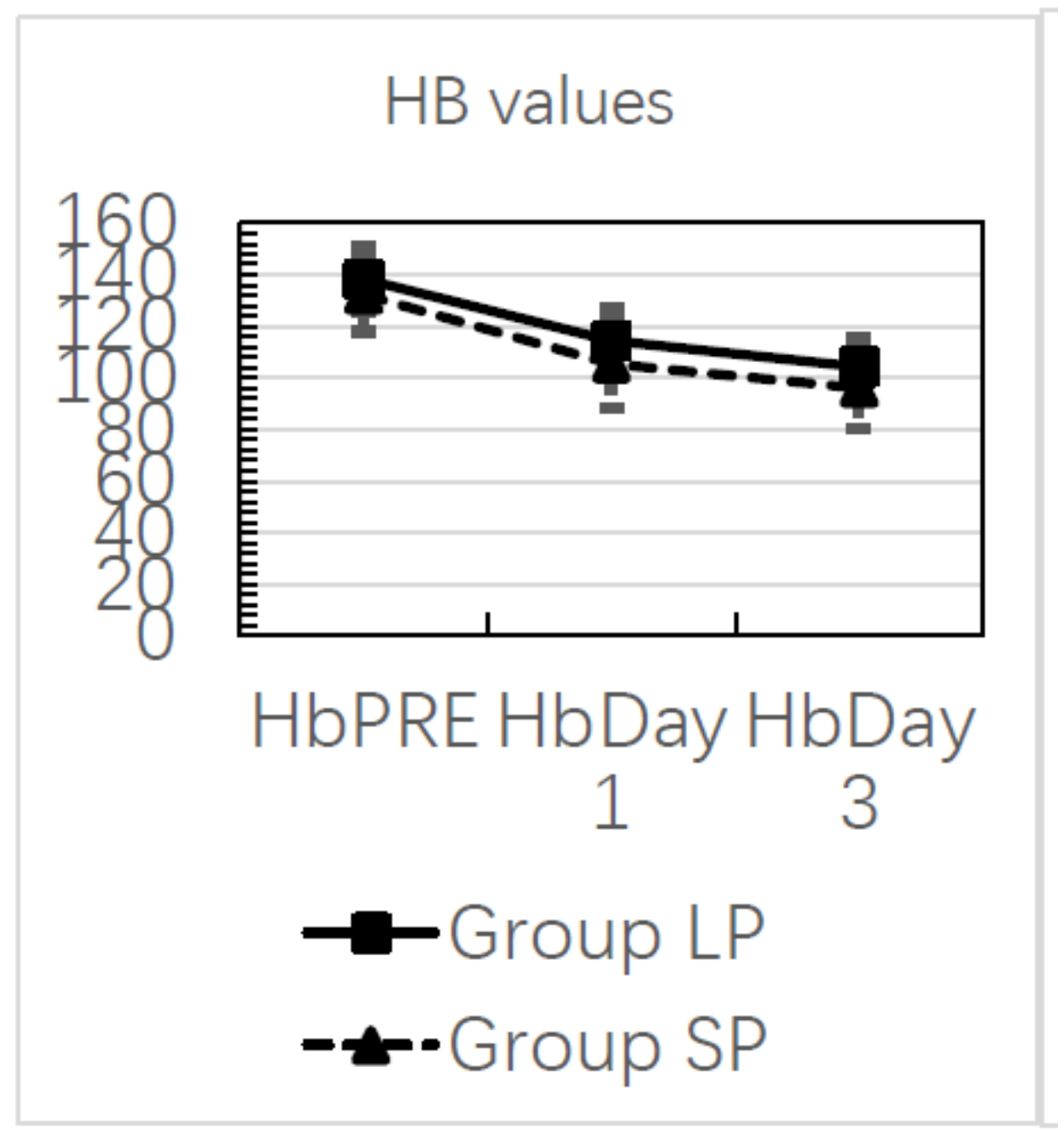

\section{Figure 1}

The course of $\mathrm{Hb}$ as a function of time for the group LP (black squares) and group SP (black triangles).The standard deviation is indicated by error bars, positive for group LP and negative for group SP. 


\section{CK-MB values}
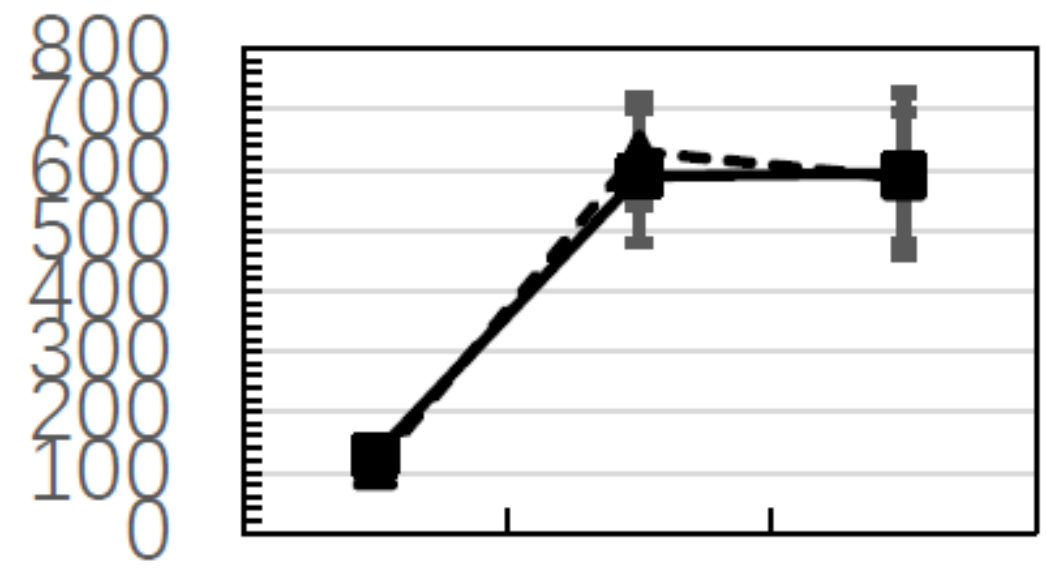

CKMB CKMB CKMB PRE Day 1 Day 3 $\rightarrow$ Group LP -๘-Group SP

Figure 2

The course of CK-MB as a function of time for the group LP (black squares) and group SP (black triangles). The standard deviation is indicated by error bars, positive for group LP and negative for group SP.

\section{Supplementary Files}

This is a list of supplementary files associated with this preprint. Click to download.

- table.pdf 